\title{
Sindezmoz yaralanmalarında güncel yaklaşımlar
}

\author{
Current concepts in syndesmotic injuries
}

\author{
Uğur Gönç, Mesut Atabek
}

LIV Hospital, Ortopedi ve Travmatoloji Kliniği, Ankara

\begin{abstract}
Ayak bileği sindezmoz yaralanmaları, ayak bileği kırıkları ile ya da nadiren izole olarak görülebilen yaralanmalardır. Eklemi ilgilendiren bu yaralanmaların anatomik redüksiyonu gereklidir. Klasik tespit yöntemi vida tespitidir. Vida tespiti ile ilgili geçmişten günümüze gelen tartışmalı birçok konu vardır. Vida tespiti farklı şekillerde yapılabilir; 3,5 mm vida ile $4,5 \mathrm{~mm}$ vida kullanımı arasında önemli bir fark tespit edilememiştir. Benzer şekilde, vidanın üç ya da dört korteks tutmasının sonuçlar üzerinde anlamlı bir etkisi yoktur. Vida çıkarılmasını rutin olarak önerenler olduğu gibi, gerekmediğini belirtenler de vardır. İmplant çıkarılmasını gerektirmediği ve daha dinamik bir tespit sağladığı düşünüldüğü için, son zamanlarda düğme dikiş implantlarının kullanımı artmıştır. Ameliyat sonrası bilgisayarlı tomografi değerlendirmelerinde malredüksiyon oranının yüksek oranda görülmesi, tespit sonrası redüksiyonun nasıl değerlendirilmesi gerektiği konusunu gündeme getirmiştir.

İzole sindezmoz yaralanmaları nadir görülür. Gizli ya da açık instabilite varlığında cerrahi tedavi endikasyonu vardır. Yüksek ayak bileği burkulması olarak adlandırılan bu yaralanma, cerrahi tedavi gerektiren olguların tanınmasındaki zorluk nedeni ile önemlidir. İnstabilite olmayan olgularda konservatif tedavi süresi diğer ayak bileği burkulmalarına göre daha uzundur.
\end{abstract}

Anahtar sözcükler: sindezmoz yaralanması; cerrahi tedavi; vida tespiti; düğme dikiş tespiti
Syndesmotic injuries usually occur after ankle fractures, but they can also be seen as isolated injuries. Anatomical reduction is mandatory in these intraarticular injuries. Although classical treatment for syndesmotic injuries is screw fixation, there are many controversial topics about this method. In the literature no difference was found between 3.5 and $4.5 \mathrm{~mm}$ screws and between three or four cortex fixation. There is also no concensus about the necessity of screw removal. In recent years suture button implants have become more commonly used for these injuries. These implants have the theoretical advantage of dynamic fixation without any need of implant removal. Another topic of discussion is the evaluation of the reduction after fixation of syndesmotic injuries since high ratio of malreduction has been detected at postoperative computerized tomography investigations.

Isolated syndesmotic injuries are relatively rare. These injuries are usually referred as high ankle sprain. Patients with occult or frank instability require surgical treatment but the diagnosis of these patients is usually difficult. In patients without instability the duration of conservative treatment is usually longer than other ankle sprains.

Key words: syndesmotic injury; surgical treatment; screw fixation; suture button fixation

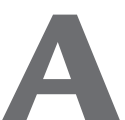
yak bileği kırıkları, alt ekstremitenin en sık görülen kırıklarıdır. Bu kırıkların yaklaşık \%23'üne, ayak bileği stabilitesinde önemli katkısı olan sindezmozun yaralanmaları da eşlik etmektedir. ${ }^{[1]}$ Sindezmoz yaralanmasının ayak bileği burkulmalarından sonra \%5-10 oranında görüldüğü bildirilmiştir. ${ }^{[2]}$ Sindezmoz yaralanmalarına yaklaşımda birçok yenilik olmasına rağmen, tedavinin nasıl yapılacağına dair tartışmalar halen devam etmektedir.

Sindezmoz, tibia ve fibulayı birbirine sıkıca bağlayarak talotibial eklemde mortisi oluşturur. Talar stabilitede aynı zamanda deltoid bağın da önemli payı mevcuttur. Distal tibiofibular eklem, konveks distal fibula ve konkav distal lateral tibia yüzeylerinden oluşan, artiküler kıkırdağı olmayan sindezmotik bir eklemdir. Eklemi ilgilendiren bu yaralanmaların anatomik redüksiyonu gereklidir. İyi tedavi edilmeyen yaralanmalar instabiliteye, sindezmozun genişlemesine ve talar kaymaya yol açar. Anatomik redüksiyon yapılmayan ve yeterli tespit yapılmamış olguların fonksiyonel sonuçları kötü olmaktadır. Bu olgularda posttravmatik osteoartritin gelişmesi kaçınılmazdır. Biyomekanik olarak yapılan

- İletişim adresi: Uzman Dr. Uğur Gönç, LIV Hospital Ankara, Bestekar Sok. No: 8, Kavaklıdere, Ankara Tel: 0532 - 3430325 e-posta: ugurgonc@hotmail.com

- Geliş tarihi: 3 Mayıs $2016 \quad$ Kabul tarihi: 3 Mayıs 2016 

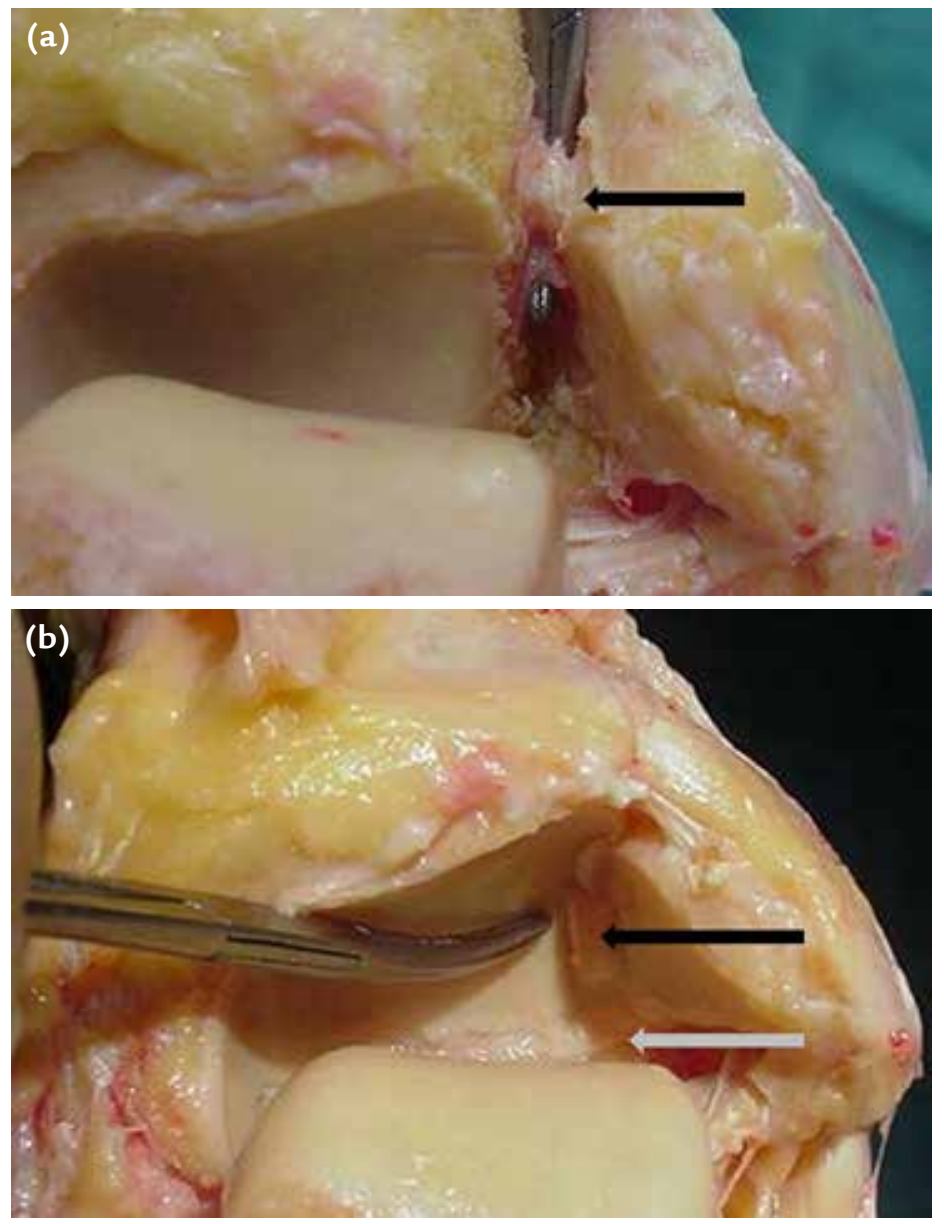

Şekil 1. a, b. Anterior inferior tibiofibular bağın anatomik görünümü (a, siyah ok). İnterosseöz bağ (siyah ok) ve inferior transvers bağın (gri ok) anatomik görünümü (b).(Çankaya Ortopedi Grubu arşivinden alınmıştır.)

ayrıntılı çalışmalarda sindezmotik bağların yaralanması sonrası aksiyel yüklenme ile özellikle dış rotasyonda, tibiotalar temas basıncının önemli ölçüde arttığı gözlenmiştir. Ayrıca, biyomekanik olarak talusun $1 \mathrm{~mm}$ laterale kaymasının, yüklenmeyi \%42 oranında arttırdığı gösterilmiştir. ${ }^{[3,4]}$

\section{ANATOMI VE YARALANMA MEKANIZMASI}

Sindezmoz dört farklı yapıdan oluşur:

1. Anterior inferior tibiofibular bağ (AiTFB) (Şekil 1a).

2. İnterosseöz bağ (IOB) (Şekil 1b).

3. Posterior inferior tibofibular bağ (PiTFB).

4. İnferior transvers bağ (iTB) (Şekil 1b).

Diastaza dirençte PITFB ve ITB \%40-45, AiTFB \%35 ve interosseöz membran $\% 35$ oranında katkı sağlar. ${ }^{[5]}$
Sindezmoz yaralanmaları çoğunlukla ayak bileğinin dış rotasyon ve hiperdorsifleksiyon mekanizması ile zorlanması sonrası oluşur. Dış rotasyon yaralanmaları ayak bileği supinasyonda ya da pronasyonda iken olabilir. Sindezmotik bağların yaralanması genellikle pronasyon - dış rotasyon mekanizması ile oluşan supra-sindezmotik Weber Tip C kırıklarda (Şekil 2) ya da Maisonneuve tipi yaralanmalar sonrası (Şekil 3a) görülür. Ancak nadir olarak, intra-sindezmotik WeberTip B ya da infra-sindezmotik WeberTip A kırıklar sonrası da sindezmoz yaralanabilir. Tip B ve Tip C kırıklarda, bazı durumlarda fibula kırı̆gı tespiti sonrasında sindesmoz stabil kalabilir. ${ }^{[6,7]}$ Biyomekanik çalışmalarda, mediyal yaralanmanın olmadığı durumlarda lateral malleol kırıklarında sindesmoz yaralansa bile talusun merkezde ve stabil kalabileceği gösterilmiştir. ${ }^{[8]}$ Stabil sindezmoz yaralanması olarak adlandırılan bu tip yaralanmalarda anterior sindezmoz yaralanması ve proksimal fibula kırığı görülür, posterior malleol ise sağlamdır. ${ }^{[9]}$ 


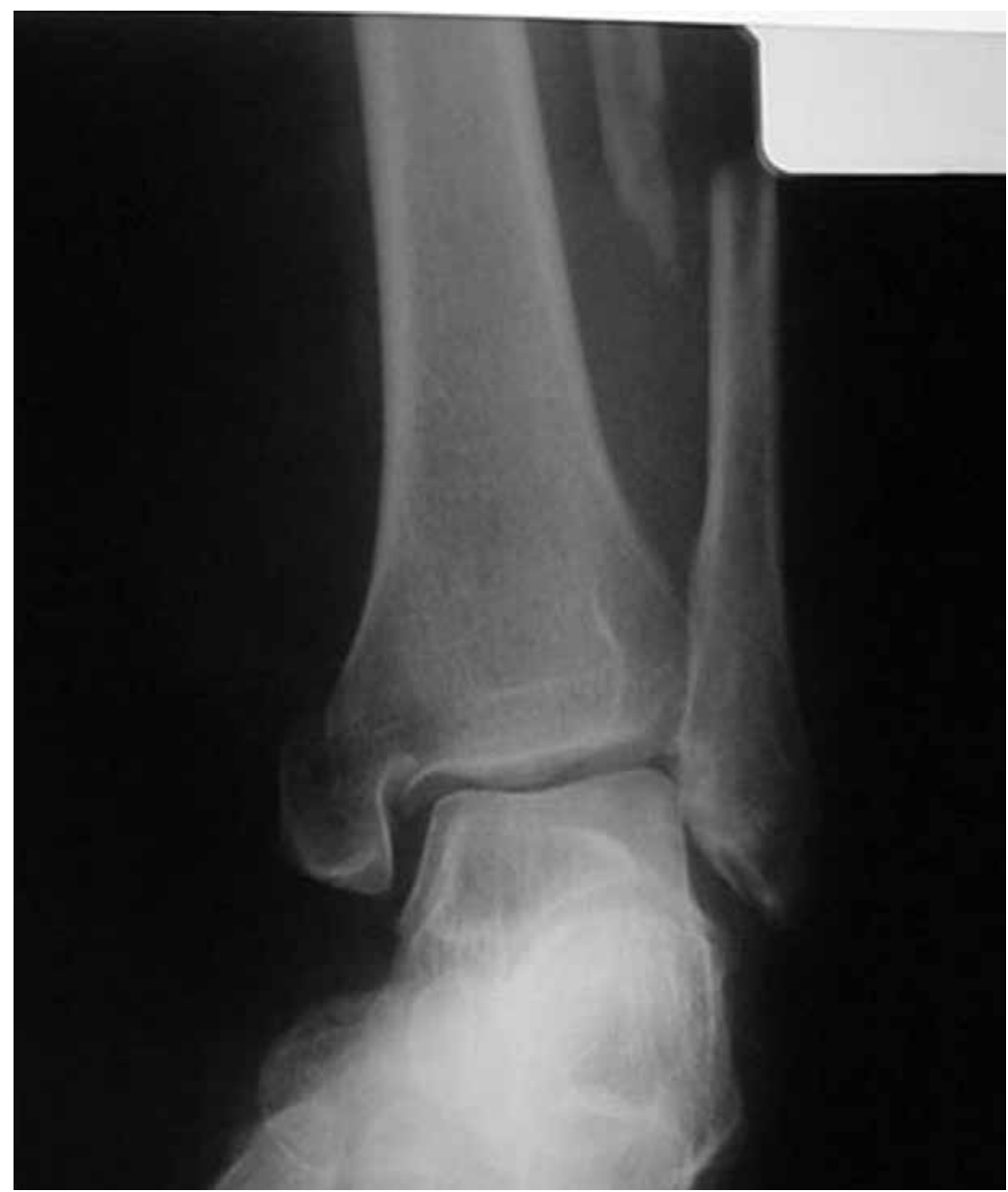

Şekil 2. Weber Tip C kırık ile beraber görülen sindezmoz yaralanması.

Sindezmoz yaralanması, bağ yırtığı olmadan, AITFB'ın tibia anterolateral köşesindeki yapışmaya yerinde (Chaput tüberkülü) veya fibuladaki yapışma yerinde (Wagslaffe tüberkülü) kopma kırığı şeklinde de oluşabilir.

Benzer mekanizmalarla, kırık oluşmadan da izole sindezmoz yaralanmaları meydana gelebilir. Talus çıkıklarında da sindezmoz yaralanması olabileceğini göz önünde bulundurmak gereklidir.

\section{RADYOLOJi}

Ayak bileği yaralanmalarında, lateral malleol kırığının seviyesi ve ek mediyal yaralanma (mediyal malleol kırığı ya da deltoid bağ yırtığı) varlığı, sindezmoz yaralanmasında önemli ipuçlarıdır. Radyolojik değerlendirme için önemli üç parametre vardır:

1. Tibiofibular örtüşme: Ön-arka grafide $10 \mathrm{~mm}$ 'den, iç rotasyon - mortis grafisinde $1 \mathrm{~mm}$ 'den fazla olmalıdır. (Şekil 4a)
2. Tibiofibular aralık: Ön-arka grafide 5 mm'den az olmalıdır. (Şekil 4b)

3. Mediyal aralık: Talar dome ile tibial plafond arasıyla eşit olmalı, 4 mm'den fazla olmamalıdır. (Şekil 4c)

Şüpheli durumlarda diğer tarafla karşılaştırmalı film çekilebilir. Dış rotasyon stres grafileri ya da basarken çekilen grafiler, bariz olmayan yaralanmalarda kullanılır. İzole yaralanmalarda, sindesmoza lokal anestezik uygulaması sonrası basarak grafiler çekilebilir. Tanıda bilgisayarlı tomografi (BT) veya manyetik rezonans (MR) görüntüleme de kullanılabilir.

Kırıklarla olan yaralanmalarda, malleol tespiti sonrası intraoperatif stres testi (çengel testi) ile sindesmoz yaralanması araştııılır. Bu test tüm kırıklar sonrası yapılmalıdır. Sindesmoz, atipik olarak beklenmeyen kırıklarda da yaralanmış olabilir. Lateral malleolün bir çengel yardımıyla laterale çekilmesi sırasında $2 \mathrm{~mm}$ 'den fazla hareket olması ve anteroposterior plandaki 

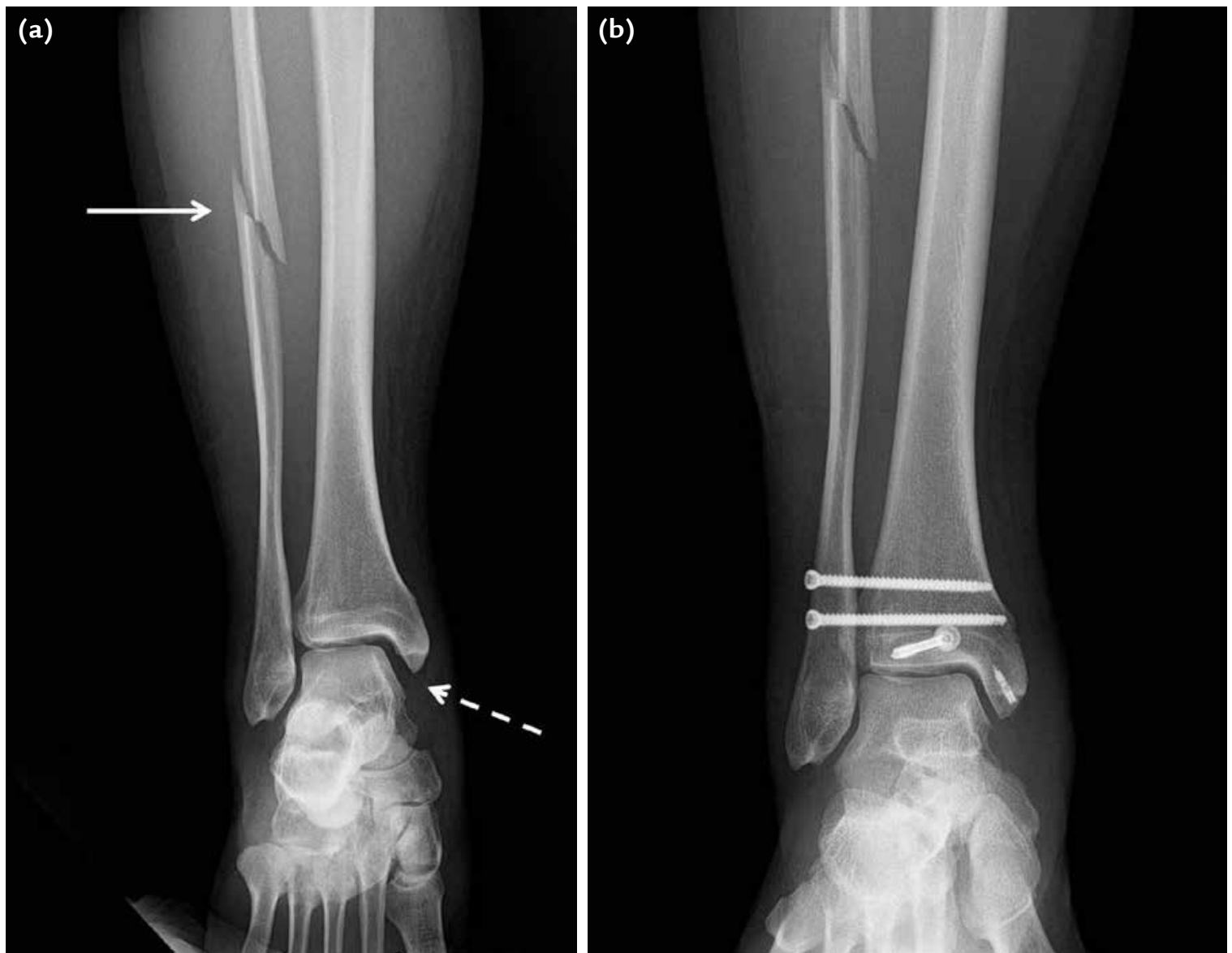

Şekil 3. a, b. Maisonneuve kırığı (deltoid bağ yırtığı ve proksimal fibula kırı̆̆ı) ile beraber görülen sindezmoz yaralanması (a). Dört korteks tutan iki adet vida ile Maisonneuve kırığında sindezmoz tespiti (b). Ayrıca deltoid bağ tamiri ve posterior malleol tespiti uygulanmış.

instabilite, testin pozitif olduğu gösterir. Kırık tespiti sonrası dış rotasyon zorlaması ile skopi görüntüsünde mediyal aralığın açılması sindesmoz yaralanmasını gösterir, ancak bu test ile bazı yaralanmalar tespit edilemeyebilir. ${ }^{[10]}$

\section{VIDA TESPITI}

Anatomik redüksiyon gerektiren bu yaralanmaların altın standart tedavisi vida tespitidir. Vidanın, eklem hattına paralel ve posteriordan anteriora doğru $30^{\circ}$ oblik gönderilmesi önerilmektedir. Vidanın çapı, kaç korteks tutulması gerektiği, sayısı, konumu, vidalama sırasında ayak bileği pozisyonu birçok soruyu ortaya çıkarırken, redüksiyonun nasıl değerlendirileceği, vidanın çıkarılıp çıkarılmayacağı, ne zaman çıkarılması gerektiği gibi ek sorular tartışmayı derinleştirmektedir.

íki adet 4,5 mm vida, mekanik olarak daha güçlü bir tespit sağlar. ${ }^{[11]}$ iki delikli kilitli plak vida tespiti,
4,5 mm vida ve dört korteks tutulumundan daha güçlüdür; 3,5 ve $4,5 \mathrm{~mm}$ 'lik vidalar aksiyel ve rotasyonel yüklenmede benzer güce sahiptir. ${ }^{[11]}$ Benzer şekilde üç ya da dört korteks tutulması karşılaştırıldığında, biyomekanik olarak aralarında anlamlı bir fark bulunmamıştır. ${ }^{[12]}$ Klinik çalışmalar da bu bulguyu desteklemektedir (Şekil 5. a, b). ${ }^{[13]}$

Bir diğer soru, vidanın tibiotalar ekleme uzaklı̆̆ıdır. Bir bilgisayarlı modelleme çalışmasında, biyomekanik olarak avantajlı seviyenin, tibiotalar eklem seviyesinin 30-40 mm proksimali olduğu bildirilmiştir. ${ }^{[14]}$ Bu çalışmayı destekleyen bir olgu serisinde, vidanın 41 mm'nin üzerinde konumlandırılması, sonuçları negatif olarak etkilemiştir. ${ }^{[15]}$ Ancak, tespitin en çok eklemin $2 \mathrm{~cm}$ üzerinde olması gerektiğini öne sürenler olduğu gibi[16], $5 \mathrm{~cm}$ 'nin altında yapılan tespitin sindezmoza daha çok yük bindirdiğini bildiren yayınlar da mevcuttur. ${ }^{[17]}$

Vida yerleştirilirken, ayak bileğinin dorsifleksiyonda olması önerilmektedir. Plantar fleksiyonda 

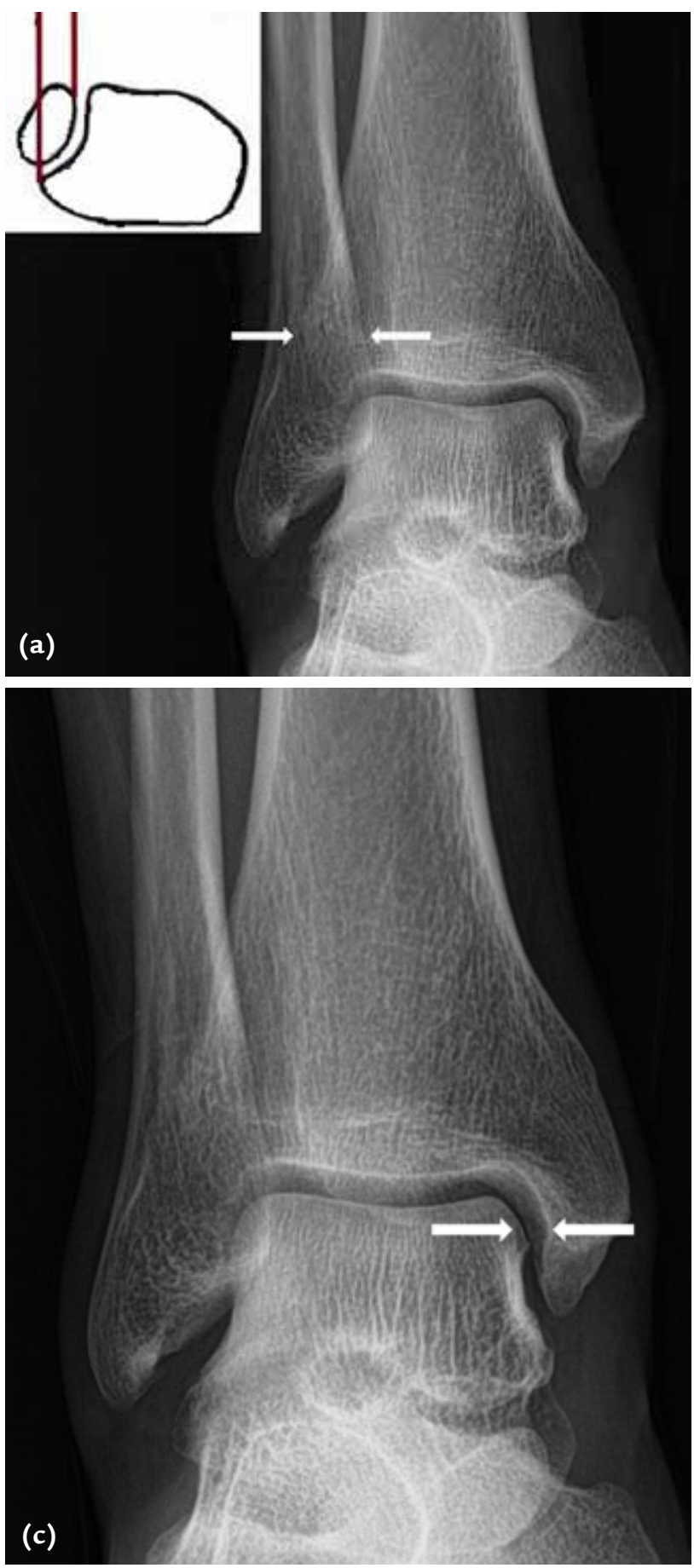

vidalama, talus ön kısmının daha geniş olması nedeniyle, sindezmozun ve mortisin daralmasına neden olarak ayak bileği hareketlerini olumsuz etkileyebilir. Genel kabul gören bu yönteme karşın, bir kadavra çalışmasında, sindesmoz vidası uygulanırken, ayak bileği pozisyonunun (dorsifleksiyon ya da plantar

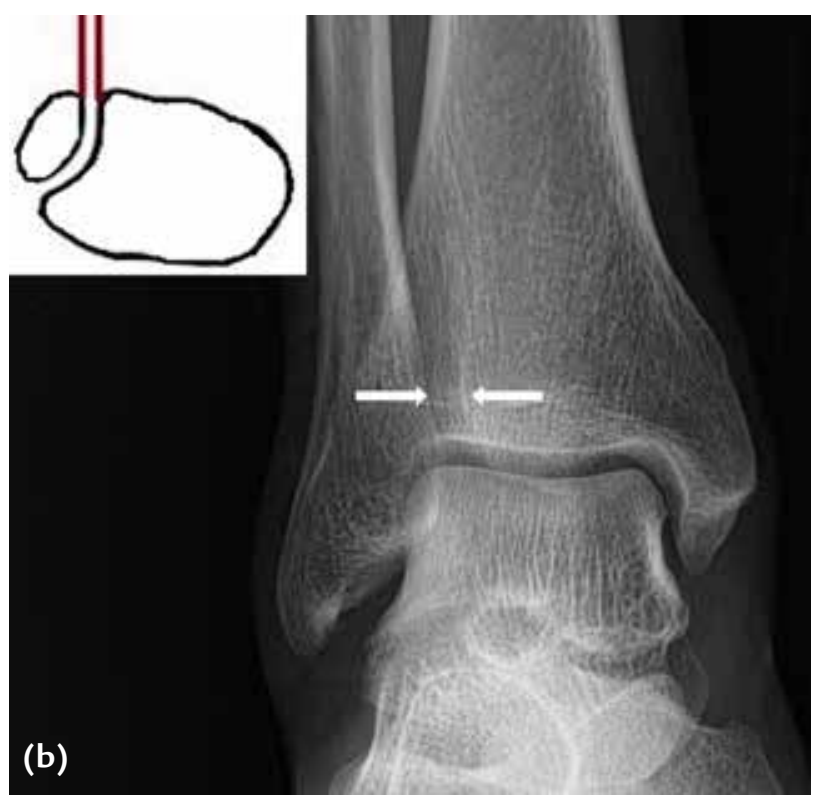

Şekil 4. a-c. Tibia posterolateral kenarı ve fibula anteromediyal kenarı arasındaki tibiofibular örtüşme ölçümü (a). Tibia anterolateral kenarı ve fibula anteromediyal kenarı arasındaki tibiofibular aralık ölçümü (b). Mediyal aralık ölçümü (c).

fleksiyon) uygulama sonrasında eklem hareketlerine etkisi olmadığı, önemli olanın anatomik redüksiyon olduğu gösterilmiştir ${ }^{[18]}$

Maisonneuve tipi pronasyon dış rotasyon yaralanmalarında, daha sağlam olan iki adet $4,5 \mathrm{~mm}$ vida ile tespit önerilmektedir. Fibula kırı̆gı direkt redükte edilmeden uzunluk ve rotasyon sağlanmalı ve sindezmoz öyle tespit edilmelidir (Şekil 3b). Bu tip yaralanmalarda, sadece bir adet vida ile tespit edilenler ile iki adet vida tespiti yapılanlar karşılaştırıldığında, bir vida ile tespitin yeterli klinik sonuçlara sahip olduğu gösterilse de, güvenli yöntem iki vida kullanılmasıdır. ${ }^{6,19]}$

\section{Malredüksiyon}

Kötü redükte edilmiş sindesmoz fonksiyonel sonuçları olumsuz etkiler. Sindesmoz vidasının düzgün konumlanması ve stabiliteyi sağlaması hasta sonuçlarını iyileştirmektedir. Son zamanlarda, literatürde iyatrojenik malredüksiyonun öneminden bahsedilmektedir. Beklenenin üstünde bir malredüksiyon varlığı nedeniyle redüksiyonun değerlendirilmesi ile ilgili çalışmalar artmıştır. Translasyonel, rotasyonel ve aşırı kompresyona bağlı malredüksiyon görülebilir. Direkt grafi ile değerlendirme yanıltıcı olabilmektedir. Postoperatif BT değerlendirme ile \%50'lere varan oranda uygun olmayan redüksiyon saptanan çalışmalar mevcuttur. ${ }^{[20]}$ Kimi yazarlara göre, malredüksiyonun hangi hastada 

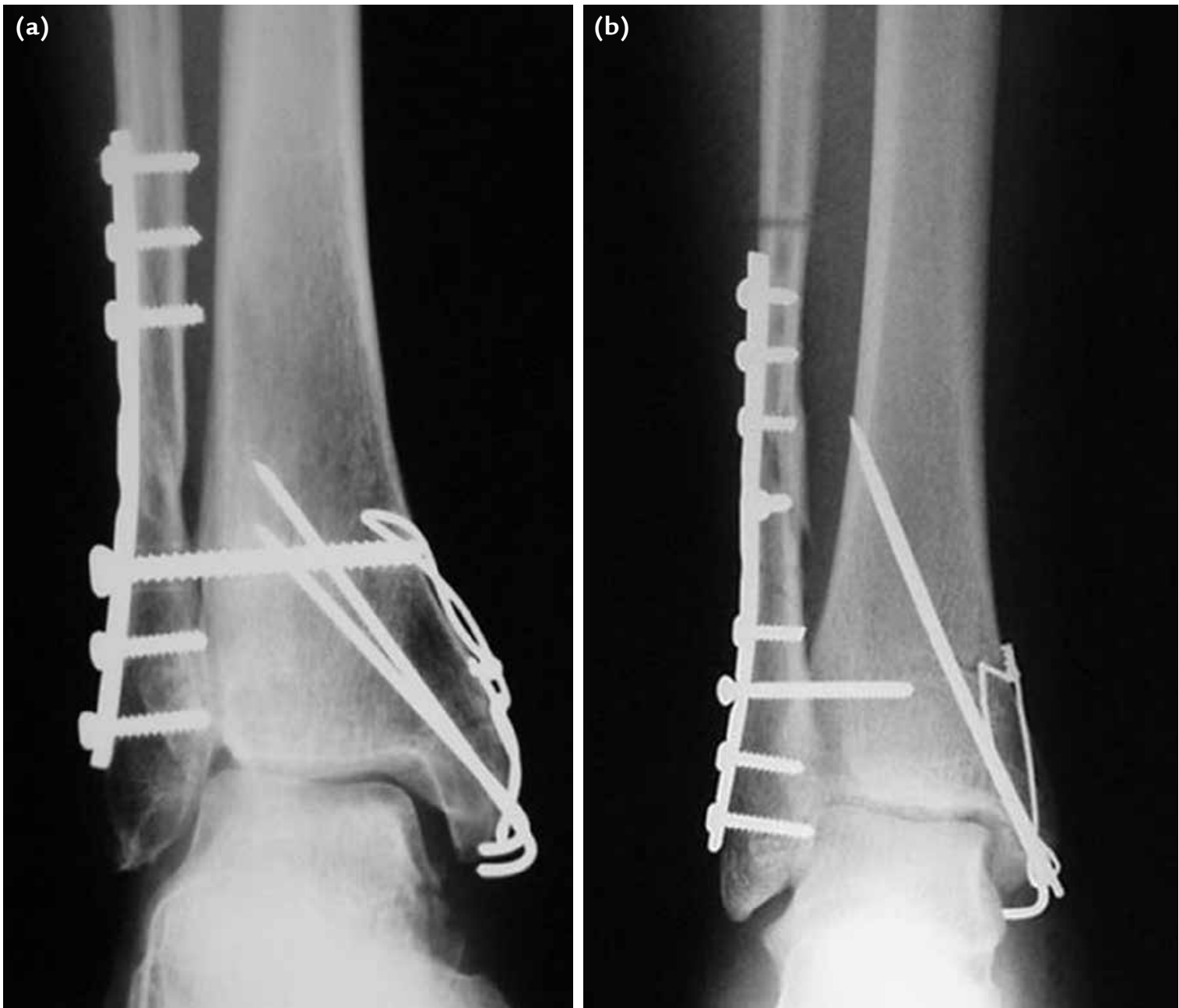

Şekil 5. a, b. Dört korteks tutan $4,5 \mathrm{~mm}$ vida ile sindezmoz tespiti (a). Üç korteks tutan $3,5 \mathrm{~mm}$ vida ile sindezmoz tespiti (b).

olabileceğini tahmin etmek güçtür. Bu nedenle, rutin intraoperatif veya postoperatif BT ile değerlendirme önerilmektedir (Şekil 6). ${ }^{[21,22]}$

Kadavra çalışmalarında, redüksiyonun klemp ile $15-30^{\circ}$ açıda yapılmasının dış rotasyona ve aşırı sıkmaya neden olabileceği gösterilirken, klemp konumlanmasının nötral anatomik aksta olmasının daha iyi redüksiyon sağladığı tespit edilmiştir. ${ }^{[23]}$ Sindezmoz vidasının aşırı sıkılması (over-compresion) ayak bileği hareketlerini ve dolayısıyla tedavinin sonuçlarını etkileyebilir. ${ }^{[20,24]}$

Her ne kadar malredüksiyonun değerlendirilmesi için çoğunlukla postoperatif BT kullanılsa da, floroskopik görüntülerin değerlendirmede yeterli olduğu, BT kontrolünün ya da sindezmozun direkt görülerek redüksiyonunun gereksiz olduğunu savunan yazarlar da mevcuttur. ${ }^{[25]}$
Posterior malleol kırıklarının eşlik ettiği yaralanmalarda, sindezmoz redüksiyonuna ayrıca dikkat etmek gerekir. Sindezmozun posteriorundaki bu tip kırıklarda, aşırı vida sıkılması sindezmozda rotasyonel malredüksiyona neden olabilir. ${ }^{[26]}$ Posterior malleolün posterior yaklaşımla tespit edildiği kırıklarda, sindezmozun direkt görülerek tespit edilebilmesi bir avantaj olabilir. Bu yaklaşımla, hastalarda malredüksiyon daha az görülmüştür. ${ }^{[27]}$

Obez hastalarda vidanın dört korteks tutması önerilmektedir. ${ }^{[28]}$ Beden-kitle endeksi yüksek hastalarda, tespit yetmezliği ve malredüksiyon daha sık görülür. ${ }^{[29]}$

\section{Vida Çıkarılması}

Tibiofibular ve tibiotalar fizyolojik hareketin engellendiği düşünülerek, vida çıkarılması önerilmektedir. Aynı zamanda, kııımış vidaların çıkarılması daha zor 


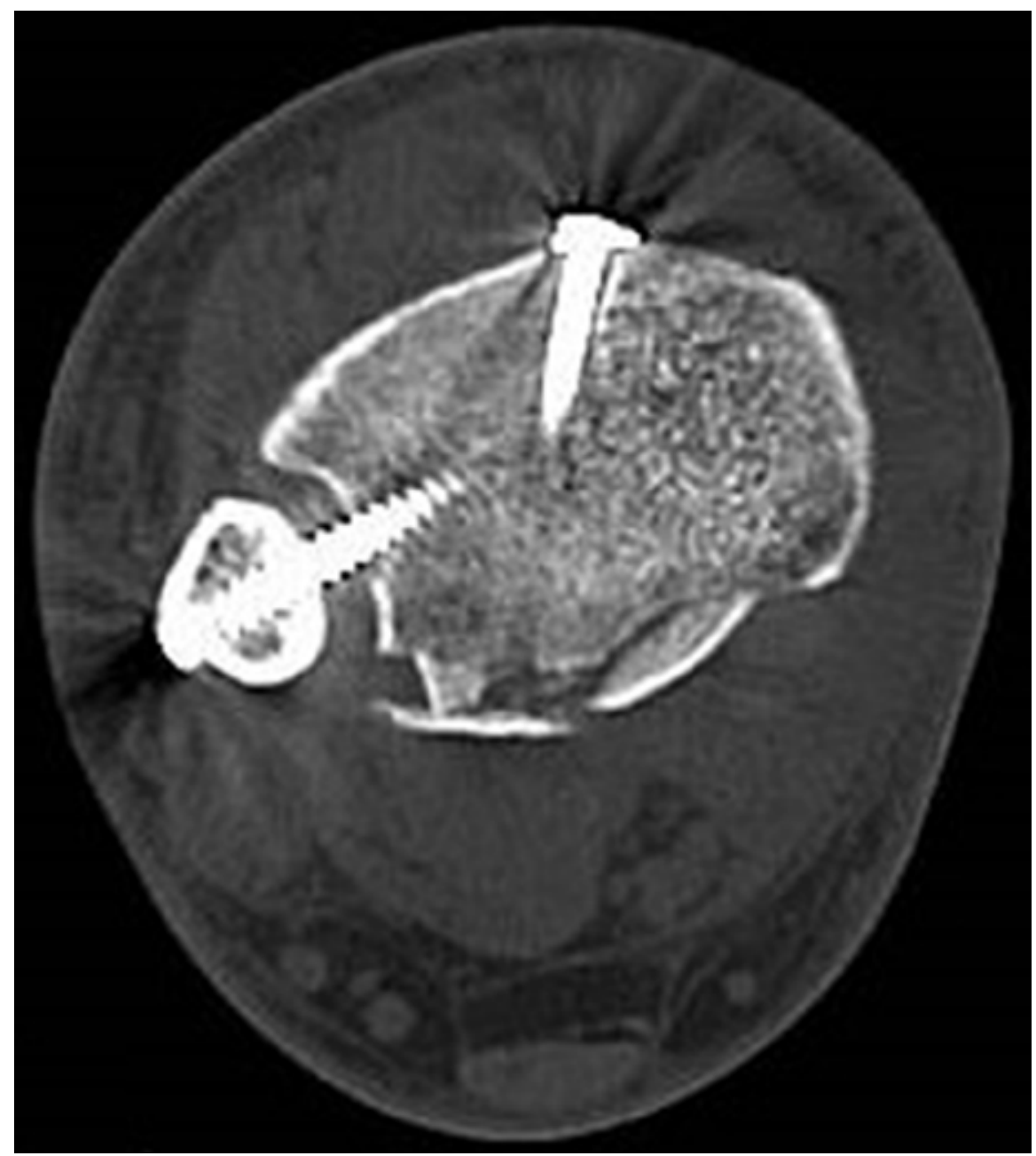

Şekil 6. Postoperatif BT ile sindezmoz redüksiyonunun kontrolü.

olmaktadır. Vidanın bağlar iyileşmeden çıkarılması ise diastaza neden olabilir. Bu nedenle, vidalar sekiz haftadan önce çıkarılmamalıdır; genel olarak 8-12 hafta arasında çıkarılması önerilmektedir. ${ }^{[6]}$ Bir kadavra çalışmasında, sindezmotik vida tespitinin tibiofibular eklem hareketlerini kısıtladığı, bu yüzden klinikte çıkarılması gerektiği savunulmuştur. ${ }^{[30]}$ Klinik başka bir çalışmada, postoperatif malredüksiyonu olan hastaların vidalarının çıkarılmasından bir ay sonra çekilen BT'lerinde, malredüksiyonun spontan düzeldiği gösterilmiştir. ${ }^{[31]}$ Bu çalışmaların bulguları vida çıkarılmasını desteklemektedir.

Bu bulgulara rağmen, vida çıkarılmasının fonksiyonel ve radyolojik sonuçlar üzerinde etkisi olmadığı birçok çalışmada gösterilmiştir. Bir çalışmada, ilginç olarak, vidaların kırıldığı grupta klinik skorlar daha yüksek bulunmuştur. ${ }^{[32]}$ Kimi yazarlara göre ,vida çıkarılmasının sonuçlar üzerinde bir etkisini yoktur; sadece semptomatik olanlar çıkarılmalıdır. ${ }^{[6,32-34]}$

Sindezmoz vidası kimi zaman 3-6 ay arasında kırılır ve bu arada sindezmoz iyileşmiştir. Vida kırılması ile sindezmoz redüksiyon kaybı arasında bir ilişki bulunamamıştır; klinik sonuçlara etkisizdir ${ }^{[35]} ; 4,5 \mathrm{~mm}$ vidalar daha az kırılır. Dört korteks uygulanan vidalarda kırılma daha fazla görülür. Çoğu zaman vidalar kırılmaz, gevşer. Bu durum, erken yük verilen veya vidanın geç çıkarıldığı hastalarda görülür. Hastaların bir kısmında, sindezmozda kalsifikasyon da görülebilir. ${ }^{[6,35]}$

Sindezmoz yaralanmaları sonrası heterotrofik ossifikasyon görülebilir. Bu durum sindezmotik sinostoza neden olarak, ağrıya ve anormal ayak bileği kinematiğine yol açabilir. Patofizyolojisi iyi anlaşılamamıştır. Sporcularda, izole yaralanmalarda ve özellikle propriyosepsiyonun bozulduğu tekrarlayıcı burkulmalarda daha sık gözlenir. ${ }^{[36]}$ 
Bütün bunların yanında, ek olarak implant çıkarılmasının ekonomik yükü vardır. ${ }^{[37]}$ Vida çıkarılmasından kaçınmak için biyobozunur vidalar kullanılabilir. Biyobozunur vida, metalik implant kadar yeterli tespit sağlar, ancak yüksek oranda yabancı cisim reaksiyonu vardır ve daha çok heterotrofik ossifikasyon görülmektedir. ${ }^{[38-40]}$

\section{DÜĞME DiKiş iMPLANTLARI}

Erken kontrollü hareket ve yüklenme, bağ fibrillerinin reorganizasyonunu ve bağın daha az skar ile iyileşmesini sağlar. Bu da bağın gücünü arttırır. Düğme dikiş implantların, dinamik stabilizasyon sağlayarak bağların daha fizyolojik iyileşmesini sağladığı düşünülmektedir.

Kadavra çalışmalarında, bu implantlarının sağladığı dinamik redüksiyonun malredüksiyonu azaltabileceği öne sürülmüştür. ${ }^{[41]}$ Biyomekanik olarak da vida ve düğme dikiş implantları arasında fark bulunmamıştır. ${ }^{[42]}$

Düğme dikiş tekniği ile tespit edilen sindesmoz yaralanmalarında, fonksiyonel skorlar vida tespitine benzer olsa da, dorsifleksiyon ve plantar fleksiyon anlamlı olarak daha iyi bulunmuştur. ${ }^{[43]}$ Benzer randomize kotrollü bir çalışmada, iki yıllık takip sonucunda radyolojik ve klinik bir fark bulunamamıştır. Malredüksiyon oranı, anlamlı olmasa da vida grubunda biraz daha yüksek bulunmuştur. ${ }^{[4]}$ Bu implantlarda vidaya göre daha az reoperasyon oranı vardır; klinik ve radyolojik olarak daha iyi sonuçlara sahip olduklarına dair yayınlar da mevcuttur. ${ }^{[45]}$ Bu teknik; fizyolojik redüksiyon sağlaması, daha erken bastırmaya izin vermesi, daha az implant problemleri oluşturması nedeniyle daha üstün bir tedavi olarak önerilmektedir. Bu tekniğin avantajları olmasına rağmen literatürde yayımlanmış, enfeksiyon, yumuşak doku irritasyonu, düğmenin gömülmesi, implant deliğinden kırıklar gibi komplikasyonlar da mevcuttur. ${ }^{[46]}$ Mediyalde yüzeyel damar sinir yaralanma riski mevcuttur (safen ven 4,5 mm uzaklıktadır); bundan kaçınmak için mediyal insizyon kullanımı öneren yazarlar vardır. ${ }^{[47]}$

\section{IZOLE SINDESMOZ YARALANMALARI}

Ayak bileği burkulmalarının bir kısmı, "yüksek ayak bileği burkulmaları" olarak adlandırılan sindezmotik yaralanmalardır. Bu yaralanmalar, ayak bileği kırığı olmadan oluşan bağ ve yumuşak doku yaralanmalarını ve tibiofibular avulsiyon kırıklarını içerir. Ayak bileği lateral burkulmalarına göre tedavisi daha uzun sürer ve sorunlara açıtır. Spora dönüş, burkulmalara göre iki kat daha uzun olmaktadır. ${ }^{[48]}$

İzole sindezmoz yaralanmaları için tanımlanmış birçok sınıflandırma sistemi vardır. En son tanımlanan ve
MR değerlendirmesine dayanan sisteme göre (Sikka Sınıflandırması); Evre 1 izole AiTFB, Evre 2 AiTFB, iOB ve intraosseöz membran (IM), Evre 3 AiTFB, IOB, iM ve PiTFB, Evre 4 bunlarla beraber deltoid bağ yaralanmasını içerir. ${ }^{[49]}$ Sınıflandırma sistemlerinde küçük farklılıklar olsa da, temelde yaralanmanın klinik yansıması benzerdir. Evre 1'de instabilite ve diastaz yoktur, Evre 3'te belirgin instabilite ve diastaz vardır. Evre 1'de konservatif tedavi başarilıken Evre 3 ve 4'te kesin cerrahi endikasyon mevcuttur. Evre 2'de gizli instabilite ve belirgin olmayan diastaz vardır. Cerrahi tedavi önerilmekle beraber tanı konulması zordur. Yaralanmanın zamanına göre; akut (altı haftadan önce), subakut (altı hafta - üç ay), kronik (üç aydan sonra) olarak sınıflandırılması, tedavi yönteminin seçimi açısından önemlidir. ${ }^{[50]}$ Erken tanı önemlidir ve yeterli tedavi, skar dokusuna bağlı sıkışma, kronik instabilite, heterotrofik ossifikasyon ve deformite gelişmeden önce planlanmalıdır. ${ }^{[5,51,52]}$

Tanıda, fizik muayene yöntemlerinin önemli payı vardır. Bir çok test tanımlanmıştır: Cotton testi (tibia distali sabitlenirken ayağa laterale doğru güç uygulanır, talusun laterale translasyonu testin pozitif olduğunu gösterir), dorsifleksiyon-kompresyon testi, eksternal rotasyon stres testi, fibular translasyon testi, palpasyon ve sıkıştırma (squeeze) testi. Ancak, bunların hepsinin duyarlılık oranları değişkendir; bir kısmı güvenilir değildir. Tüm bu tanı çabalarına rağmen, fizik muayene ile izole yaralanmalar $\% 20$ oranında tespit edilememektedir. ${ }^{[53,54]}$

En son yayımlanan ESSKA-AFAS konsensusuna göre, izole sindezmoz yaralanmaları stabil ve stabil olmayan olarak ayrılmıştır. Sindezmotik rüptür ile beraber deltoid bağ rüptürü olduğu zaman, yaralanma instabil olarak değerlendirilir. Stabil yaralanmalar, kısa bacak alçı veya breysler ile tedavi edilebilir. Hasta üç hafta bastırılmaz, sonrasında propriyoseptif egzersizlere başlanır. Instabil yaralanmalar ise cerrahi olarak tedavi edilmelidir. Tanı koyarken kullanılabilecek testler: AiTFB üzerinde lokal hassasiyet, fibular translasyon testi, Cotton testleridir. Radyolojik olarak, AP grafi ve mortis grafisinde tibiofibular aralık, tibiofibular örtüşme ve mediyal aralık ölçümleri önemlidir. Stres testlerinin kullanımı, güvenilirliği düşük olduğu için önerilmemektedir. Ek tetkik olarak MR kullanılabilir. ${ }^{[55]}$

Akut yaralanmalarda tedavi, sindezmozun direkt tamiri, deltoid bağ tamiri ve vida ya da düğme dikiş tespit şeklinde yapılabilir. ${ }^{[28]}$ Subakut izole sindezmotik yaralanmalarda, bağ ilerletme ile başarılı sonuçlar alınan olgular mevcuttur. ${ }^{[56]}$ İzole kronik sindezmoz instabilitelerinde artroskopik sınıflandırma ve rekonstrüksiyon teknikleri tanımlanmıştır. AiTFB tamiri, periosteal flep ile bağ tamiri, plantaris tendon ile rekonstrüksiyon gibi 
teknikler kullanılmıştır. Bu hastalarda, preoperatif döneme göre klinik skorlar daha iyi bulunmuştur. ${ }^{[57]}$

MR ile posterior malleol kırı̆ıı olmayan instabil yaralanmalarda PITFB yaralanması gösterilmiştir. Yeni tanınmaya başlayan bu tip kırıksız posterior yaralanmalar, ilerleyen zamanlarda spesifik bir konu olarak gündeme gelebilir. ${ }^{[58]}$

Sonuç olarak, sindezmoz yaralanmaları ile ilgili birçok yenilik ve çalışmaya rağmen, tam olarak cevaplanmamış sorular yeni araştırmaları gerekli kılmaktadır. Artık, vida tespitinin altın standart olup olmadığı tartışılmaktadır. Anatomik redüksiyon gerekliliği vurgulanmakla birlikte, redüksiyonun nasıl değerlendirileceği konusunda fikir birliği yoktur. Vida çıkarılmasının gerekli olup olmadığı halen tartışmalıdır. Yeni tip implantların getirdiği yeniliklere rağmen, vida tespiti ile karşılaştırıldığında, benzer sonuçlara sahip olmaları nedeniyle belirgin bir üstünlükleri yoktur. Üstelik, düğme implantların kaç adet ve hangi seviyeden konulması gerektiği gibi yeni sorular gündeme gelecektir. Tüm bu tartışmalara rağmen, klinik yayınlarda şu ana kadar kullanılan yöntemlerin yeterli başarı oranına sahip olduğu bilinmelidir. İzole sindezmoz yaralanmalarında belirgin bir instabilite yoksa, daha dikkatli olunmalı ve gizli instabilite araştırılmalıdır.

\section{KAYNAKLAR}

1. Purvis GD. Displaced, unstable ankle fractures: classification, incidence, and management of a consecutive series. Clin Orthop Relat Res 1982;(165):91-8.

2. van Staa TP, Dennison EM, Leufkens HG, Cooper C. Epidemiology of fractures in England and Wales. Bone 2001;29(6):517-22.

3. Hunt KJ, Goeb Y, Behn AW, Criswell B, Chou L. Ankle Joint Contact Loads and Displacement With Progressive Syndesmotic Injury. Foot Ankle Int 2015;36(9):1095-103. Crossref

4. Ramsey PL, Hamilton W. Changes in tibiotalar area of contact caused by lateral talar shift. J Bone Joint Surg Am 1976;58(3):356-7.

5. Ogilvie-Harris DJ, Reed SC, Hedman TP. Disruption of the ankle syndesmosis: biomechanical study of the ligamentous restraints. Arthroscopy 1994;10(5):558-60.

6. Marsh JL, Saltzman CL, Ankle Fractures. Bucholz RW, Heckman JD, editors. Rockwood and Green's Fractures in Adults. Lippicott Williams and Wilkins; 2011. p.2147-247.

7. Kortekangas T, Flinkkilä T, Niinimäki J, Lepojärvi S, Ohtonen $\mathrm{P}$, Savola O, Pakarinen H. Effect of syndesmosis injury in SER IV (Weber B)-type ankle fractures on function and incidence of osteoarthritis. Foot Ankle Int 2015;36(2):180-7. Crossref

8. Brown TD, Hurlbut PT, Hale JE, Gibbons TA, Caldwell NJ, Marsh JL, Nepola JV. Effects of imposed hindfoot constraint on ankle contact mechanics for displaced lateral malleolar fractures. J Orthop Trauma 1994;8(6):511-9.

9. Harris IA, Jones HP. The fate of the syndesmosis in type $C$ ankle fractures: a cadaveric study. Injury 1997;28(4):275-7.
10. Pakarinen $H$, Flinkkilä $T$, Ohtonen $P$, Hyvönen $P$, Lakovaara $M$, Leppilahti J, Ristiniemi J. Intraoperative assessment of the stability of the distaltibiofibular joint in supinationexternal rotation injuries of the ankle: sensitivity, specificity, and reliability of two clinical tests. J Bone Joint Surg Am 2011;93(22):2057-61. Crossref

11. Xenos JS, Hopkinson WJ, Mulligan ME, Olson EJ, Popovic NA. The tibiofibular syndesmosis. Evaluation of the ligamentous structures, methods of fixation, and radiographic assessment. J Bone Joint Surg Am 1995;77(6):847-56.

12. Markolf KL, Jackson SR, McAllister DR. Syndesmosis fixation using dual $3.5 \mathrm{~mm}$ and $4.5 \mathrm{~mm}$ screws with tricortical and quadricortical purchase: a biomechanical study. Foot Ankle Int 2013;34(5):734-9. Crossref

13. Karapinar $\mathrm{H}$, Kalenderer $\mathrm{O}$, Karapinar L, Altay $\mathrm{T}$, Manisali $\mathrm{M}$, Gunal I. Effects of three- or four-cortex syndesmotic fixation in ankle fractures. J Am Podiatr Med Assoc 2007;97(6):457-9.

14. Verim O, Er MS, Altinel L, Tasgetiren S. Biomechanical evaluation of syndesmotic screw position: a finite-element analysis. J Orthop Trauma 2014;28(4):210-5. Crossref

15. Schepers $T$, van der Linden $H$, van Lieshout EM, Niesten DD, van der Elst M. Technical aspects of the syndesmotic screw and their effect on functional outcome following acute distal tibiofibular syndesmosis injury. Injury 2014;45(4):775-9. Crossref

16. McBryde A, Chiasson B, Wilhelm A, Donovan F, Ray T, Bacilla P. Syndesmotic screw placement: a biomechanical analysis. Foot Ankle Int 1997;18(5):262-6.

17. Miller RS, Weinhold PS, Dahners LE. Comparison of tricortical screw fixation versus a modified suture construct for fixation of ankle syndesmosis injury: a biomechanical study. J Orthop Trauma 1999;13(1):39-42.

18. Tornetta P 3rd, Spoo JE, Reynolds FA, Lee C. Overtightening of the ankle syndesmosis: is it really possible? J Bone Joint Surg Am 2001;83-A(4):489-92.

19. Lambers KT, van den Bekerom MP, Doornberg JN, Stufkens $\mathrm{SA}$, van Dijk CN, Kloen P. Long-term outcome of pronationexternal rotation ankle fractures treated with syndesmotic screws only. Bone Joint Surg Am 2013;95(17):e1221-7. Crossref

20. Gardner MJ, Graves ML, Higgins TF, Nork SE. Technical Considerations in the Treatment of Syndesmotic Injuries Associated With Ankle Fractures. J Am Acad Orthop Surg 2015;23(8):510-8. Crossref

21. Gardner MJ, Demetrakopoulos D, Briggs SM, Helfet DL, Lorich DG. Malreduction of the tibiofibular syndesmosis in ankle fractures. Foot Ankle Int 2006;27(10):788-92.

22. Franke J, von Recum J, Suda AJ, Vetter S, Grützner PA, Wendl $K$. Predictors of a persistent dislocation after reduction of syndesmotic injuries detected with intraoperative threedimensional imaging. Foot Ankle Int 2014;35(12):1323-8. Crossref

23. Miller AN, Barei DP, laquinto JM, Ledoux WR, Beingessner DM. latrogenic syndesmosis malreduction via clamp and screw placement. J Orthop Trauma 2013;27(2):100-6. Crossref

24. Phisitkul P, Ebinger T, Goetz J, Vaseenon T, Marsh JL. Forceps reduction of the syndesmosis in rotational ankle fractures: a cadaveric study. J Bone Joint Surg Am 2012;94(24):2256-61. Crossref

25. Summers HD, Sinclair MK, Stover MD. A reliable method for intraoperative evaluation of syndesmotic reduction. J Orthop Trauma 2013;27(4):196-200. Crossref 
26. Cherney SM, Haynes JA, Spraggs-Hughes AG, McAndrew CM, Ricci WM, Gardner MJ. In Vivo Syndesmotic Overcompression After Fixation of Ankle Fractures With a Syndesmotic Injury. J Orthop Trauma 2015;29(9):414-9. Crossref

27. Miller AN, Carroll EA, Parker RJ, Boraiah S, Helfet DL, Lorich DG. Direct visualization for syndesmotic stabilization of ankle fractures. Foot Ankle Int 2009;30(5):419-26. Crossref

28. van Dijk CN, Longo UG, Loppini M, Florio $P$, Maltese L, Ciuffreda M, Denaro V. Conservative and surgical management of acute isolated syndesmotic injuries: ESSKAAFAS consensus and guidelines. Knee Surg Sports Traumatol Arthrosc 2016;24(4):1217-27. Crossref

29. Mendelsohn ES, Hoshino CM, Harris TG, Zinar DM. The effect of obesity on early failure after operative syndesmosis injuries. J Orthop Trauma 2013;27(4):201-6. doi: 10.1097/ BOT.0b013e31825cf921

30. Huber $\mathrm{t}$, Schmoelz W, Bölderl A. Motion of the fibula relative to the tibia and its alterations with syndesmosis screws: A cadaver study. Foot Ankle Surg 2012;18(3):203-9. Crossref

31. Song DJ, Lanzi JT, Groth AT, Drake M, Orchowski JR, Shaha $\mathrm{SH}$, Lindell KK. The Effect of Syndesmosis Screw Removal on the Reduction of the Distal Tibiofibular Joint: A Prospective Radiographic Study. Foot Ankle Int 2014;35(6):543-8.

32. Kaftandziev I, Spasov M, Trpeski S, Zafirova-Ivanovska B, Bakota B. Fate of the syndesmotic screw -Search for a prudent solution. Injury 2015;46 Suppl 6:S125-9. Crossref

33. Tucker A, Street J, Kealey D, McDonald S, Stevenson M. Functional outcomes following syndesmotic fixation: A comparison of screws retained in situ versus routine removal -Is it really necessary? Injury 2013;44(12):1880-4. Crossref

34. Boyle MJ, Gao R, Frampton CM, Coleman B. Removal of the syndesmotic screw after the surgical treatment of a fracture ofthe ankle in adult patients does not affect oneyear outcomes: a randomised controlled trial. Bone Joint J 2014;96-B(12):1699-705. Crossref

35. Stuart K, Panchbhavi VK. The fate of syndesmotic screws. Foot Ankle Int 2011;32(5):S519-25. Crossref

36. Van Heest TJ, Lafferty PM. Injuries to the ankle syndesmosis. J Bone Joint Surg Am 2014;96(7):603-13. Crossref

37. Lalli TA, Matthews LJ, Hanselman AE, Hubbard DF, Bramer MA, Santrock RD. Economic impact of syndesmosis hardware removal. Foot (Edinb) 2015;25(3):131-3. Crossref

38. Sun $H$, Luo CF, Zhong B, Shi HP, Zhang CQ, Zeng BF. A prospective, randomised trial comparing the use of absorbable and metallic screws in the fixation of distal tibiofibular syndesmosis injuries: mid-term follow-up. Bone Joint J 2014;96-B(4):548-54. Crossref

39. Böstman OM. Distal tibiofibular synostosis after malleolar fractures treated using absorbable implants. Foot Ankle 1993;14(1):38-43.

40. Taylor DC, Englehardt DL, Bassett FH 3rd. Syndesmosis sprains of the ankle. The influence of heterotopic ossification. Am J Sports Med 1992;20(2):146-50.

41. Westermann RW, Rungprai C, Goetz JE, Femino J, Amendola A, Phisitkul P. The effect of suture-button fixation on simulated syndesmotic malreduction: a cadaveric study. J Bone Joint Surg Am 2014;96(20):1732-8. Crossref

42. Gough BE, Chong AC, Howell SJ, Galvin JW, Wooley PH. Novel flexible suture fixation for the distal tibiofibular syndesmotic joint injury: a cadaveric biomechanical model. J Foot Ankle Surg 2014;53(6):706-11. Crossref
43. Seyhan M, Donmez F, Mahirogullari M, Cakmak S, Mutlu S, Guler O. Comparison of screw fixation with elastic fixation methods in the treatment of syndesmosis injuries in ankle fractures. Injury 2015;46 Suppl 2:S19-2. Crossref

44. Kortekangas T, Savola O, Flinkkilä T, Lepojärvi S, Nortunen S, Ohtonen $\mathrm{P}$, Katisko J, Pakarinen $\mathrm{H}$. A prospective randomised study comparing TightRope and syndesmotic screw fixation for accuracy and maintenance of syndesmotic reduction assessed with bilateral computed tomography. Injury 2015;46(6):1119-26. Crossref

45. Laflamme M, Belzile EL, Bédard L, van den Bekerom MP, Glazebrook M, Pelet S. A prospective randomized multicenter trial comparing clinical outcomes of patients treated surgically with a static or dynamic implant for acute ankle syndesmosis rupture. J Orthop Trauma 2015;29(5):216-23. Crossref

46. Hong CC, Lee WT, Tan KJ. Osteomyelitis after TightRope ${ }^{\circledR}$ fixation of the ankle syndesmosis: a case report and review of the literature. J Foot Ankle Surg 2015;54(1):130-4. Crossref

47. Pirozzi KM, Creech CL, Meyr AJ. Assessment of Anatomic Risk During Syndesmotic Stabilization With the Suture Button Technique. J Foot Ankle Surg 2015;54(5):917-9. Crossref

48. Lin CF, Gross ML, Weinhold P. Ankle syndesmosis injuries: anatomy, biomechanics, mechanism of injury, and clinical guidelines for diagnosis and intervention. J Orthop Sports Phys Ther 2006;36(6):372-84.

49. Sikka RS, Fetzer GB, Sugarman E, Wright RW, Fritts $H$, Boyd JL, Fischer DA. Correlating MRI findings with disability in syndesmotic sprains of NFL players. Foot Ankle Int 2012;33(5):371-8. Crossref

50. Valkering KP, Vergroesen DA, Nolte PA. Isolated syndesmosis ankle injury. Orthopedics 2012;35(12): e1705-10. Crossref

51. Bonnin JG. Injury to the ligaments of the ankle. J Bone Joint Surg Br 1965;47(4):609-11.

52. Nussbaum ED, Hosea TM, Sieler SD, Incremona BR, Kessler DE. Prospective evaluation of syndesmotic ankle sprains without diastasis. Am J Sports Med 2001;29(1):31-5.

53. Beumer A, Swierstra BA, Mulder PG. Clinical diagnosis of syndesmotic ankle instability: evaluation of stress tests behind the curtains. Acta Orthop Scand 2002;73(6):667-9.

54. Sman AD, Hiller CE, Refshauge KM. Diagnostic accuracy of clinical tests for diagnosis of ankle syndesmosis injury: a systematic review. Br J Sports Med 2013;47(10):620-8. Crossref

55. van Dijk CN, Longo UG, Loppini $M$, Florio $P$, Maltese $L$, Ciuffreda $M$, Denaro V. Classification and diagnosis of acute isolated syndesmotic injuries: ESSKA-AFAS consensus and guidelines. Knee Surg Sports Traumatol Arthrosc 2016;24(4):1200-16. Crossref

56. Jain SK, Kearns SR. Ligamentous advancement for the treatment of subacute syndesmotic injuries. Report of a new technique in 5 cases. Foot Ankle Surg 2014;20(4):281-4. Crossref

57. Colcuc C, Fischer S, Colcuc S, Busse D, Bliemel C, Neun O, Abt HP, Hoffmann R. Teatment strategies for partial chronic instability of the distal syndesmosis: an arthroscopic grading scale and operative staging concept. Arch Orthop Trauma Surg 2016;136(2):157-63. Crossref

58. Warner SJ, Garner MR, Schottel PC, Hinds RM, Loftus ML, Lorich DG. Analysis of PITFL injuries in rotationally unstable ankle fractures. Foot Ankle Int 2015;36(4):377-82. Crossref 